\title{
EP-82
}

\section{Pure laparoscopic versus open right hepatectomy including the middle hepatic vein for living donor liver transplantation}

\author{
Sanggyun Suh, Suk Kyun HONG*, Su Young HONG, Eui Soo HAN, Kwangpyo HONG, \\ Jeong-Moo LEE, YoungRok CHOI, Nam-Joon YI, Kwang-Woong LEE, Kyung-Suk SUH
}

Department of Surgery, Seoul National University College of Medicine, Seoul, Korea

Introduction: Pure laparoscopic donor hepatectomy (PLDH) has become increasingly accepted in the era of minimally invasive surgeries.However, the outcomes of pure laparoscopic donor right hepatectomy (PLDRH) including the middle hepatic vein (MHV) are relatively less known than for left lateral sectionectomy or right hepatectomy.

Methods: This study aimed to report our experience with and the outcomes of PLDRH including the middle hepatic vein (MHV) and to compare these outcomes with conventional donor right hepatectomy (CDRH) including the MHV. The medical records of living liver donors between January 2002 and December 2020 at Seoul National University Hospital were retrospectively.

Results: To minimize selection bias, donors who underwent CDRH including the MHV after the initiation of the PLDH program were excluded. Finally, there were 42 donors who underwent CDRH including the MHV and 37 who underwent PLDRH including the MHV. The total operation time was 284 minutes versus 250 minutes $(p=0.01)$ and estimated blood loss was $394 \mathrm{~mL}$ versus $208 \mathrm{~mL}(p=$ 0.01). There were no postoperative complications in the PLDRH including the MHV group. The rate of complications in recipients was similar in both groups.

Conclusions: PLDRH including the MHV appears to be safe and feasible. Further analysis including longterm outcomes is needed. 